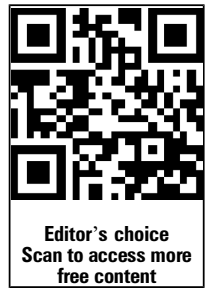

free content

\title{
Finding patients before they crash: the next major opportunity to improve patient safety
}

\author{
David W Bates, ${ }^{1,2,3}$ Eyal Zimlichman ${ }^{1,4}$
}

${ }^{1}$ Division of General Internal Medicine, The Center for Patient Safety Research and Practice, Brigham and Women's Hospital, Boston, Massachusetts, USA ${ }^{2}$ Partners Healthcare Systems, Inc, Wellesley, Boston, Massachusetts, USA

${ }^{3}$ Harvard Medical School, Boston, Massachusetts, USA

${ }^{4}$ Sheba Medical Center, Tel Hashomer, Israel

\section{Correspondence to}

Dr David W Bates, Division of General Internal Medicine,

The Center for Patient Safety Research and Practice, Brigham and Women's Hospital, 1620 Tremont St, Boston, MA 2120, USA; dbates@partners.org

Received 18 August 2014 Accepted 28 August 2014 Published Online First 24 September 2014

\section{SLinked}

- http://dx.doi.org/10.1136/ bmjqs-2014-003073

\section{CrossMark}

To cite: Bates DW,

Zimlichman E. BMJ Qual Saf 2015;24:1-3.
Adverse events cause large numbers of deaths in hospitals, and many more serious injuries. Often, however, it is difficult or impossible in a specific circumstance to determine whether or not the outcome would have been different with an earlier intervention. ${ }^{1}{ }^{2}$ On the other hand, substantial evidence exists for many conditions-sepsis for exampleshowing that earlier, more aggressive intervention can improve patient outcomes, especially with the use of protocols or guidelines. ${ }^{3}$

Better monitoring of sick patients has been a major interest in hospitals for decades and was a major rationale for the development of intensive care units (ICUs). Much of the research on monitoring comes from intensive care. Patients on general wards (ie, outside ICUs) are increasingly sick, but the level of monitoring they receive varies substantially, with intervals between manual vital signs measurement easily spanning $8-12 \mathrm{~h}$. Often patients have already decompensated to a significant extent before transfer to an ICU is contemplated.

We believe that the coming together of four major trends or innovations promises substantial improvements to patient outcomes by preventing this perennial problem of delayed recognition and management of deteriorating patients on general hospital wards. These trends include the uniform use of electronic health records in hospitals, ${ }^{4}$ major advances in physiological sensor development, ${ }^{5}$ the rapid adoption of mobile technologies, ${ }^{6}$ and the ability to perform analytics in the background to provide decision support at the point of care. ${ }^{7}$

Adoption rates for electronic health records in the USA have risen from approximately $20 \%$ to over $80 \%$ in recent years. ${ }^{8}$ Although the comprehensiveness of these records varies substantially, a high proportion include electronic vital signs, which offers major opportunities to improve the early detection and management of patients at risk of clinical deterioration. Other countries, such as the UK and Canada, currently lag behind in terms of widespread adoption of electronic health records, but this may well change soon. Even now, leading institutions have begun adopting in most industrialised nations.

New types of physiological sensors represent another breakthrough-and a staggering array are becoming available. These range from devices that sit under the mattress and can continuously detect pulse, respirations and whether or not the patient is moving ${ }^{9}$ through to endtidal $\mathrm{CO}_{2}$ monitoring to 'hightechnology' wearable garments that can detect physiological parameters ${ }^{10}$ to name just a few examples.

The 'mobile revolution'11 constitutes a key part of the 'triple revolution'-the internet, mobile technology and social networks. Use of mobile technology by clinical staff has become increasingly widespread in healthcare. In addition to facilitating rapid communication, it will become increasingly valuable as a data-entry tool and as an enabler for users to consume data.

Finally, 'big data' and analytics in general are finally coming to healthcare and offer opportunities to deliver major improvements in care delivery. ${ }^{7}$ For this area of enhanced patient monitoring, improvements will depend on harnessing and combining several types of data, but especially vital signs and clinical laboratory results, to identify patients with early signs of deterioration. The ability to identify such situations will likely be particularly enhanced when trends and combinations of variables are examined rather than simple cutoffs. For example, 
a fall in blood pressure at the same time as an increase in pulse and a decrease in urine output is of much more concern than a fall in blood pressure alone. Combining other clinical factors, such as the presence of a known infection or recent surgery, will also have specific implications that can add to point-of-care alerts for clinical staff.

In the above context, the study by Schmidt et $a l^{12}$ represents an important milestone. The authors used an electronic patient safety surveillance system (EPSS), while leveraging the use of electronic health records, mobile technology and analytical approaches, to find patients who appeared to be decompensating. Remarkably, they found reductions in mortality for patients within 56 diagnosis groups used in the National Health Service (NHS) to assess quality from $7.8 \%$ to $6.4 \%$ at one hospital, and $7.6 \%$ to $6.2 \%$ at the other. It is remarkable that the authors achieved these reductions roughly concurrently at the two hospitals given the logistic challenges of implementing this intervention. The authors apparently did not use new sensor technologies at either institution, basing the alerting mechanism on intermittent vital signs measurement. Furthermore, the analytical approaches used do not appear to be particularly sophisticated, which suggests that even bigger improvements are probably possible. Hospital mortality has been stubbornly resistant to improvement, so the lowering of mortality at the two study hospitals reported by Schmidt et al ${ }^{12}$ represents a truly dramatic improvement.

The study and approach taken have many strengths and a number of weaknesses. The authors speculate that the EPSS may have been effective because it increased the likelihood that more complete vital signs data would be collected, enabled fast delivery of decision support, anticipated user needs, included simple data screens, asked for data only when necessary, and enabled tracking. If a user entered worrisome vital signs, they received immediate feedback, and entry was available everywhere because of the use of wireless and mobile. It is notable that they did not measure all-cause mortality, and this should be done in the future. Confounders are always an important concern, but none stand out in this case as an obvious issue. Moreover, the study did find reductions in mortality for the 56 diagnoses used to monitor performance in the NHS, and these in aggregate account for $83 \%$ of inpatient deaths in UK hospitals. That the intervention showed similar reductions in mortality at the two hospitals provides further reassurance.

Of course, many previous efforts have aimed to improve monitoring in general and detection of decompensating patients in particular. A great deal of attention and focus has been placed on rapid response teams. While the evidence to support them has been mixed, a recent meta-analysis concluded that moderate evidence supports their use. ${ }^{13}$ As with many such interventions, rapid response teams would probably be more beneficial if deteriorating patients were recognised earlier. This can be achieved by automating the identification of a deteriorating patient through continuous monitoring and use of analytics as well as directly activating the response teams so that cultural barriers are mitigated. Mobile technology can also be leveraged-for example, to push worrisome signals about decompensation to responsible caregivers so they can come to evaluate the patient.

Continuous monitoring can be achieved through the use of continuous pulse oximetry, which is now routinely used at many hospitals to monitor many types of patients, especially after surgery. ${ }^{14}{ }^{15}$ End-tidal capnography shows promise for detecting respiratory compromise earlier than oximetry might. ${ }^{16}$ Another approach that brings together multiple technologies is the EarlySense technology. ${ }^{9}$ This brings together: a sensor that detects pulse, respiratory rate and whether or not the patient is moving; real-time analytical tools to detect false-positive warnings ${ }^{17}$; and modern communication mobile technology to communicate the results to the responsible provider. In a recent study, ${ }^{9}$ this approach reduced the length of stay of patients in general medical-surgical intervention units and the number of subsequent ICU days for patients in intervention units, although it did not significantly affect the likelihood of transfer to the ICU. Moreover, it appeared highly cost-effective. ${ }^{18}$

The results of the study by Schmidt et $a l^{12}$ have a number of implications. Before the approach is implemented widely, it would be helpful if the results could be validated prospectively in at least a few more hospitals. But the intervention is simple, and it should be possible to spread it widely within the NHS, which would have a major effect.

More broadly, it is also clear that recognition of decompensation should receive a great deal of attention going forward. Future research should address the relative benefits and cost-effectiveness of different approaches, and this should be publicly supported. In addition, research should target different clinical settings -ICUs, general care units, long-term care and high-risk patients at home. The false-positive issue has to be addressed-alert fatigue is the rule in this area. The greatest benefits are likely to be realised first in general care units-this is likely to be the 'lowest-hanging' fruit, although we believe there will also very likely be substantial improvement for high-risk patients at home.

Overall, the use of more effective monitoring approaches promises to reduce mortality rates substantially for hospital patients. The most successful interventions will probably bring together a variety of technologies-electronic health records, sensors, mobile devices and analytics. But, to implement these interventions effectively in the complex environments in healthcare, we will have to pay careful attention to sociotechnical factors, as they can trump even the best technologies. 
Acknowledgements The authors thank Betty Liu for her assistance with the preparation of this manuscript.

Contributors DWB planned, conceptualised the manuscript, drafted the article and gave final approval of the version to be submitted. EZ conceptualised the manuscript, participated in the drafting and revising the article and gave final approval of the submitted version.

Competing interests DWB and EZ both serve on the clinical advisory board of, and have received research support from, EarlySense, which makes devices that can detect patient decompensation.

Provenance and peer review Not commissioned; internally peer reviewed.

\section{REFERENCES}

1 Hogan H, Healey F, Neale G, et al. Preventable deaths due to problems in care in English acute hospitals: a retrospective case record review study. BMJ Qual Saf 2012;21:737-45.

2 Hayward RA, Hofer TP. Estimating hospital deaths due to medical errors: preventability is in the eye of the reviewer. JAMA 2001;286:415-20.

3 van Zanten AR, Brinkman S, Arbous MS, et al. Guideline bundles adherence and mortality in severe sepsis and septic shock. Crit Care Med 2014;42:1890-8.

4 Adler-Milstein J, Everson J, Lee SY. Sequencing of EHR adoption among US hospitals and the impact of meaningful use. J Am Med Inform Assoc 2014;21:984-91.

5 Pol MC, Poerbodipoero S, Robben S, et al. Sensor monitoring to measure and support daily functioning for independently living older people: a systematic review and road map for further development. J Am Geriatr Soc 2013;61:2219-27.

6 Fiordelli M, Diviani N, Schulz PJ. Mapping mHealth research: a decade of evolution. J Med Internet Res 2013;15:e95.

7 Bates DW, Saria S, Ohno-Machado L, et al. Big data in health care: using analytics to identify and manage high-risk and high-cost patients. Health Aff (Millwood) 2014;33:1123-31.
8 Adler-Milstein J, Jha AK. No evidence found that hospitals are using new electronic health records to increase medicare reimbursements. Health Aff (Millwood) 2014;33:1271-7.

9 Brown H, Terrence J, Vasquez P, et al. Continuous monitoring in an inpatient medical-surgical unit: a controlled clinical trial. Am J Med 2014;127:226-32.

10 Seoane F, Ferreira J, Alvarez L, et al. Sensorized garments and textrode-enabled measurement instrumentation for ambulatory assessment of the autonomic nervous system response in the ATREC project. Sensors (Basel) 2013;13:8997-9015.

11 Rainie H, Wellman B. Networked: the new social operating system. Cambridge, Mass: MIT Press, 2012.

12 Schmidt PE, Meredith P, Prytherch DR, et al. Impact of introducing an electronic physiological surveillance system on hospital mortality. BMJ Qual Saf 2015;24:10-20.

13 Winters BD, Weaver SJ, Pfoh ER, et al. Rapid-response systems as a patient safety strategy: a systematic review. Ann Intern Med 2013;158(5 Pt 2):417-25.

14 Curry JP, Jungquist CR. A critical assessment of monitoring practices, patient deterioration, and alarm fatigue on inpatient wards: a review. Patient Saf Surg 2014;8:29.

15 Taenzer AH, Pyke JB, McGrath SP, et al. Impact of pulse oximetry surveillance on rescue events and intensive care unit transfers: a before-and-after concurrence study. Anesthesiology 2010;112:282-7.

16 Nagler J, Krauss B. Capnography: a valuable tool for airway management. Emerg Med Clin North Am 2008;26:881-97, vii.

17 Zimlichman E, Szyper-Kravitz M, Shinar Z, et al. Early recognition of acutely deteriorating patients in non-intensive care units: assessment of an innovative monitoring technology. J Hosp Med 2012;7:628-33.

18 Slight SP, Franz C, Olugbile M, et al. The return on investment of implementing a continuous monitoring system in general medical-surgical units*. Crit Care Med 2014;42:1862-8. 